\title{
Antimicrobial Resistance And Molecular
}

\section{Characteristics Among Neisseria gonorrhoeae Clinical Isolates In A Chinese Tertiary Hospital}

This article was published in the following Dove Press journal: Infection and Drug Resistance

\author{
Zhou Zheng' \\ Li Liu' \\ Xiaofei Shen' \\ Jingyi Yu' \\ Lijiang Chen' \\ Lingling Zhan' \\ Han Chen' \\ Chunchan Lin' \\ Ye Jiang \\ Hong Xia' \\ Liangxing Wang ${ }^{2}$ \\ Fangyou Yu ${ }^{1,3}$ \\ 'Department of Clinical Laboratory, The \\ First Affiliated Hospital of Wenzhou \\ Medical University, Wenzhou, Zhejiang, \\ People's Republic of China; ${ }^{2}$ Department \\ of Respiratory Medicine, The First \\ Affiliated Hospital of Wenzhou Medical \\ University, Wenzhou 325000, People's \\ Republic of China; ${ }^{3}$ Department of \\ Laboratory Medicine, Shanghai \\ Pulmonary Hospital, Tongji University \\ School of Medicine, Shanghai 200443, \\ People's Republic of China
}

Purpose: The resistance of $N$. gonorrhoeae to antimicrobial agents has been increasing year by year due to the overuse of antibiotics. The primary aims of the present study were to investigate the molecular characteristics of the clinical isolates of Neisseria gonorrhoeae and the resistance to azithromycin in a Chinese tertiary hospital.

Methods: From January 2014 to May 2017, a total of 55 clinical isolates of N. gonorrhoeae were collected. Genes associated with azithromycin resistance (AZM-R), including mutations in 23S rRNA alleles, the $m t r R$ promoter and coding regions, and $r p l D$ and $r p l V$ were evaluated by PCR and DNA sequencing. All clinical isolates were subjected to N. gonorrhoeae multiantigen sequence typing (NG-MAST), while the AZM-R isolates were further characterized by multilocus sequence typing (MLST).

Results: The AZM-R rate in this study was $23.64 \%$ (13/55), and a single (A)-nucleotide deletion mutation in the $m t r R$ promoter region, a G45D mutation in the $m t r R$ coding region, a point mutation in $r p l D$, and an A2047G mutation in 23S rRNA alleles were detected in 13, 4, 3 and 4 isolates, respectively; no mutations were found in $r p l V$. There was no significant difference in the $m t r R$ coding region mutation rate between the azithromycin-sensitive and AZM-R groups $(P>0.05)$; however, there was a significant difference in the mutation rate of the $m t r R$ promoter region $(P<0.05)$. Among the 55 isolates studied, 43 distinct NG-MAST were determined, while the AZM-R isolates were allocated into 10 distinct MLST/NGMAST combinations. All three isolates with high-level AZM-R belonged to the sequence types (STs) NG-MAST ST1866 and MLST ST10899.

Conclusion: N. gonorrhoeae clinical isolates from Wenzhou, eastern China, showed considerable genetic diversity. Measures should be implemented to monitor the spread of the NG-MAST ST1866 and MLST ST10899 N. gonorrhoeae clones, which exhibit high-level AZM-R in eastern China.

Keywords: Neisseria gonorrhoeae, azithromycin, antimicrobial resistance, NG-MAST, MLST

\section{Introduction}

Department of Clinical Laboratory, The First Affiliated Hospital of Wenzhou Medical University, Wenzhou, Zhejiang, People's Republic of China

Email wzjxyfy@I63.com

Liangxing Wang

Department of Respiratory Medicine, The

First Affiliated Hospital of Wenzhou

Medical University, Wenzhou 325000,

People's Republic of China

Email 38805@I63.com
Gonorrhea is the second most common sexually transmitted bacterial infection in the world. According to a recent estimation of WHO, the number of gonococcal infections occurred worldwide reached 78.3 million cases in the year 2012. ${ }^{1}$ Antimicrobial resistance (AMR) of $N$. gonorrhoeae has become a major public health problem worldwide. In recent years, azithromycin has been increasingly used for the treatment of gonorrhea, and the resistance rate of $N$. gonorrhoeae to azithromycin has risen alongside this elevated use. ${ }^{2,3}$ In many countries, the sporadic development of 
high-level azithromycin resistance (AZM-R) and numerous clinical failures have led to great concern and threaten the long-term efficacy of the currently recommended approach for treatment of gonorrhea. ${ }^{4-6}$

Multidrug resistance of $N$. gonorrhoeae is related to the overexpression of efflux pumps. The most important efflux mechanism is the MtrC-MtrD-MtrE system, encoded by the $m t r$ operon, in which $m t r R$ is a regulatory gene and $m \operatorname{tr} C D E$ are the structural genes. ${ }^{7}$ Another efflux pump, encoded by the mef gene, was originally identified in some of the gram-positive organisms and has since also been discovered in clinical strains of $N$. gonorrhoeae. ${ }^{8}$

The ribosomal proteins, L4 (encoded by $r p l D$ ) and L22 (encoded by $r p l V$ ), bind to domain I of 23S rRNA and act as channels, through which macrolide antibiotics enter the ribosome. Point mutations in $r p l D$ and $r p l V$, conferring resistance to macrolides, have been described in Escherichia coli, ${ }^{9}$ and in rplD for Streptococcus pneumoniae; ${ }^{10}$ however, such mutations are rarely detected in N. gonorrhoeae. ${ }^{11}$ In contrast, 23S rRNA point mutations have been described, including C2611T (numbering refers to the E. coli genome), conferring low-to-moderate levels of AZM-R (minimum inhibitory concentrations (MICs) $=2$ to $32 \mu \mathrm{g} / \mathrm{mL}$ ) or A2059G (E. coli numbering), conferring high-level AZM-R (MICs $\geq 256 \mu \mathrm{g} / \mathrm{mL}$ ). ${ }^{12}$

As a commonly used molecular typing method, $N$. gonorrhoeae multiantigen sequence typing (NG-MAST) detects nucleotide sequence variations in both $\operatorname{por} B$ and tbp $B$ and determines the sequence types (STs) of isolates. ${ }^{13}$ A single $N$. gonorrhoeae can rapidly diversify into multiple NG-MAST STs by the introduction of mutations or recombinational replacement events. ${ }^{14}$ Thus, the evaluation of NG-MAST STs is useful for short-term epidemiology. Over longer periods of time, rapid diversification can conceal the fact that several NG-MAST STs may be minor variants of the same $N$. gonorrhoeae strain. ${ }^{15}$

Multilocus sequence typing (MLST) is used to characterize isolates by amplifying sequences from seven housekeeping loci and is commonly used to track the spread of $N$. gonorrhoeae strains, where indexed genetic variation accumulates over longer periods of time. ${ }^{13}$

Little is known for types of AZM-R gonococci currently circulating in China until 2013; hence, levels of AZM-R and the molecular characteristics of AZM-R N. gonorrhoeae remain unclear. ${ }^{6,11,16,17}$ In the absence of new antimicrobial agents for the treatment of gonorrhea, the classification and evaluation of the dynamics of AZM-R N. gonorrhoeae strains on a regional and national basis are important for successful review of treatment recommendations. The resistance levels of $N$. gonorrhoeae to azithromycin in Wenzhou, eastern China, are unclear. The purpose of this study was to investigate the prevalence and genetic characteristics of $N$. gonorrhoeae from a teaching hospital in Wenzhou.

\section{Materials And Methods}

\section{Bacterial Strains}

From January 2014 to May 2017, a total of 55 nonrepetitive $N$. gonorrhoeae isolates were selected from 189 strains by simple random sampling in the First Affiliated Hospital of Wenzhou Medical University, of which 11 were from 2014, 10 from 2015, 17 from 2016, and 17 from 2017, where more than one sample was obtained from the same patient and only the first isolate was retained. All strains were identified by Gram-staining, oxidase reaction, and sugar fermentation tests and further confirmed as $N$. gonorrhoeae using a fully automatic rapid microbial mass spectrometry detection system (VITEK MS from bioMérieux, SA, France).

\section{Antimicrobial Susceptibility Testing}

Susceptibility of $N$. gonorrhoeae to antimicrobials, including penicillin, tetracycline, ciprofloxacin, spectinomycin, ceftriaxone, and azithromycin, was determined using the E-test method (Wenzhou Kangtai Biological Technology Co., Ltd.). E-test results were interpreted based on the Clinical and Laboratory Standards Institute (CLSI 2019) guidelines criteria for all antibiotics. ${ }^{18}$ A single $N$. gonorrhoeae isolate was streaked onto Thayer-Martin (T-M) selective medium for separation and purification. Inoculated plates were incubated at $36^{\circ} \mathrm{C}$ in $5 \% \mathrm{CO}_{2}$ for 24-48 hrs. Then, bacteria at $0.5 \mathrm{M}$ turbidity were smeared on six 9-cm diameter GC plates three times uniformly and densely and incubated for 24 to $48 \mathrm{hrs}$ at $35^{\circ} \mathrm{C}$ in a $5 \%$ $\mathrm{CO}_{2}$ atmosphere with six E-test strips to determine MIC values. All strains were preserved in glycerin broth and stored at $-80^{\circ} \mathrm{C}$ until used. The quality control strain of the antibiotic susceptibility test is $N$. gonorrhoeae ATCC 49226, which is provided by the Clinical Laboratory of the Ministry of Health of the People's Republic of China and kept by the laboratory. The strain is stored in the glycerol broth liquid medium.

\section{Extraction Of Bacterial Genomic DNA}

Genomic DNA was extracted by boiling. Briefly, 3-5 N. gonorrhoeae colonies were suspended in $200 \mu \mathrm{L}$ of sterile 
distilled water and boiled for $10 \mathrm{mins}$ at $100^{\circ} \mathrm{C}$, then centrifuged at $13,800 \mathrm{~g}$ for $15 \mathrm{mins}$ at $4^{\circ} \mathrm{C}$. Finally, supernatants were removed and stored at $-20^{\circ} \mathrm{C}$ for further use.

\section{PCR And DNA Sequencing Of Genes Associated With Azithromycin Resistance}

In an attempt to identify point mutations, sequences of genes related to AZM-R were determined, including $23 \mathrm{~S}$ rRNA alleles, $r p l D$ and $r p l V$ (encoding ribosomal proteins L4 and L22, respectively), ${ }^{19}$ and the $m t r R$ promoter and coding regions. Primers (listed in Table 1) for $m t r R$ were designed using primer premier 5.0, while those for $r p l D$ and $r p l V$ have been published previously. ${ }^{20}$

PCR conditions for the $m t r R$ promoter region were 10 mins at $94^{\circ} \mathrm{C}$ for predenaturation, followed by 30 cycles of $1 \mathrm{~min}$ at $94^{\circ} \mathrm{C}$ for denaturation, $1 \mathrm{~min}$ at $50^{\circ} \mathrm{C}$ for annealing, and extension at $72^{\circ} \mathrm{C}$ for $1 \mathrm{~min}$. PCR conditions for the $m t r R$ coding region were 10 mins at $94^{\circ} \mathrm{C}$, then 30 cycles of $1 \mathrm{~min}$ at $94^{\circ} \mathrm{C}, 45 \mathrm{~s}$ at $48^{\circ} \mathrm{C}$, and $1 \mathrm{~min}$ at $72^{\circ} \mathrm{C}$. PCR conditions for $r p l D$ were 4 mins at $94^{\circ} \mathrm{C}$, then 30 cycles of $30 \mathrm{~s}$ at $94^{\circ} \mathrm{C}, 30 \mathrm{~s}$ at $60^{\circ} \mathrm{C}$, and $45 \mathrm{~s}$ at $72^{\circ} \mathrm{C}$. Conditions for amplification of $r p l V$ were 4 mins at $94^{\circ} \mathrm{C}$, then 30 cycles of $30 \mathrm{~s}$ at $94^{\circ} \mathrm{C}, 30 \mathrm{~s}$ at $55^{\circ} \mathrm{C}$, and $1 \mathrm{~min}$ at $72^{\circ} \mathrm{C}$.

A two-step PCR method was developed to identify mutations within domain $\mathrm{V}$ of each of the four copies of the 23S rRNA gene. The first step used the Upstream-F primer, paired with a primer specific for each of the $23 \mathrm{~S}$
rRNA alleles, as previously described (Table 1). ${ }^{21}$ PCR conditions were 30 cycles of $1 \mathrm{~min}$ at $94^{\circ} \mathrm{C}, 1.5 \mathrm{mins}$ at $68^{\circ} \mathrm{C}$ (for alleles 1 and 4 ) or $66^{\circ} \mathrm{C}$ (for alleles 2 and 3), and 2.5 mins at $72^{\circ} \mathrm{C}$. The products obtained were used as a template in a second PCR using the Upstream-F and Downstream-R2 primers to amplify the four copies of the 23S rRNA gene for sequence determination. For the second PCR step, the conditions were 35 cycles of: $1 \mathrm{~min}$ at $94^{\circ} \mathrm{C}, 1 \mathrm{~min}$ at $59^{\circ} \mathrm{C}$, and $1 \mathrm{~min}$ at $72^{\circ} \mathrm{C} .{ }^{21}$

DNA samples amplified by PCR were subjected to electrophoresis at $110 \mathrm{~V}$ for 30 mins. Then, all amplified products were photographed using a gel imager and confirmed to be single bands. Next, PCR products were purified using magnetic beads (by Beijing Qingke New Industry Biotechnology Co., Ltd. Hangzhou Branch) and directly sequenced using first-generation sequencing technology. DNA sequences were aligned using BLAST and GenBank programs (http://www.ncbi.nlm.nih.gov/blast/) to identify gene mutations.

\section{N. gonorrhoeae Multiantigen Sequence Typing (NG-MAST) And Multi-Locus Sequence Typing (MLST)}

All $N$. gonorrhoeae isolates were subjected to molecular epidemiologic analysis using NG-MAST, which assigns sequence types (STs) based on a combination of two highly variable alleles ( $\operatorname{por} B$ and $t b p B$ ). Alignments of the por $B$

Table I The Resistance Gene Primer Sequences And Target Fragment Sizes

\begin{tabular}{|c|c|c|c|c|}
\hline Designation & \multicolumn{2}{|c|}{ PCR Primer Sequence $\left(5^{\prime} \rightarrow 3^{\prime}\right)$} & Product Size (bp) & Annealing Temperature $\left({ }^{\circ} \mathrm{C}\right)$ \\
\hline mtrR Promoter Region & $\begin{array}{l}\mathrm{F} \\
\mathrm{R}\end{array}$ & $\begin{array}{l}\text { GCCAATCAACAGGCATTCTTA } \\
\text { GTTGGAACAACGCGTCAAAC }\end{array}$ & 367 & 50 \\
\hline$m t r R$ coding region & $\begin{array}{l}\mathrm{F} \\
\mathrm{R}\end{array}$ & $\begin{array}{l}\text { GCACGCATCGCCTTAG } \\
\text { AGTTGTCCATCATTATCCC }\end{array}$ & 842 & 48 \\
\hline rpID & $\begin{array}{l}\mathrm{F} \\
\mathrm{R}\end{array}$ & $\begin{array}{l}\text { CAGCGATGTTGTAGTTCGT } \\
\text { ACTCAAGTAATCTTGGCGC }\end{array}$ & 482 & 60 \\
\hline rp/V & $\begin{array}{l}\mathrm{F} \\
\mathrm{R}\end{array}$ & $\begin{array}{l}\text { TCAGCGACAATATGGTTGGT } \\
\text { AGCCCAGTCTTTAGTTACC }\end{array}$ & 468 & 55 \\
\hline allele I & $\mathrm{R}$ & TCAGAATGCCACAGCTTACAAACT & 2054 & 68 \\
\hline allele 2 & $\mathrm{R}$ & GCGACCATACCAAACACCCACAGG & 2240 & 66 \\
\hline allele 3 & $\mathrm{R}$ & GATCCCGTTGCAGTGAAGAAAGTC & 2217 & 66 \\
\hline allele 4 & $\mathrm{R}$ & AACAGACTTACTATCCCATTCAGC & 1847 & 68 \\
\hline Downstream & $\mathrm{R} 2$ & TTCGTCCACTCCGGTCCTCTCGTA & 712 & 59 \\
\hline Upstream & $\mathrm{F}$ & ACGAATGGCGTAACGATGGCCACA & & \\
\hline
\end{tabular}


and $t b p B$ nucleotide sequences were modified using EditSeq software (LaserGene software version 7.1; DNAStar Corp) before submission to the NG-MAST website (www.ngmast.net) for NG-MAST STs. MEGA 7.0 software, with the maximum-likelihood method, was used to create $N$. gonorrhoeae phylogenetic trees by concatenating por $B$ and $t b p B$ alleles. ${ }^{11}$ AZM-R were further typed using MLST, for which seven housekeeping genes $(a b c Z, a d k$, aroE, fumC, $g d h, p d h$, and pgm) were analyzed and the sequencing results submitted to the database (http://pubmlst. org/neisseria/) to obtain allele numbers. Strain sequences were then obtained based on allele numbers (STs).

\section{Results}

\section{Patient Data}

Among 55 patients with gonorrhea, there were 52 males and 3 females whose age distribution range was 16-78 years (mean $\pm \mathrm{SD}, 32.0 \pm 11.2$ years); $65.5 \%$ (36/55) were married and the main diagnosis is urethritis.

\section{Antimicrobial Susceptibility}

Among the $55 \mathrm{~N}$. gonorrhoeae strains tested, 52 (94.5\%), 19 (34.5\%), 49 (90.1\%), 0 (0.0\%), 0 (0.0\%), and 13 $(23.6 \%)$ were resistant to penicillin, tetracycline, ciprofloxacin, spectinomycin, ceftriaxone, and azithromycin, respectively. MIC values for azithromycin ranged from 0.064 to $>256 \mu \mathrm{g} / \mathrm{mL}$, with an $\mathrm{MIC}_{50}$ of $0.5 \mu \mathrm{g} / \mathrm{mL}$ and an $\mathrm{MIC}_{90}$ of $1.0 \mu \mathrm{g} / \mathrm{mL}$. The rates of resistance to azithromycin for the years from 2014 to 2017 were $18.2 \%(2 / 11)$, $10.0 \%(1 / 10), 29.4 \%(5 / 17)$, and $29.4 \%$ (5/17), respectively. There were 21 multidrug resistant (MDR) strains (strains with resistance to three or more types of antimicrobial agent) among the 55 strains of $N$. gonorrhoeae, and all 13 AZM-R strains were multidrug resistant. Of the 13 AZM-R strains, all were resistant to penicillin and ciprofloxacin, five were resistant to tetracycline, and the remaining eight exhibited intermediate resistance to tetracycline. Among the 13 AZM-R isolates, eight isolates with MIC values of $1 \mu \mathrm{g} / \mathrm{mL}$ were classified as exhibiting lowlevel resistance, two with MIC values of $4 \mu \mathrm{g} / \mathrm{mL}$ showed moderate-level resistance, and three with MIC values of $>256 \mu \mathrm{g} / \mathrm{mL}$ exhibited high-level resistance.

\section{Detection Of Mutations In Genes} Associated With Azithromycin Resistance The following mutations were detected in 13, 4, and 4 of the 13 AZM-R isolates, respectively: a single-nucleotide
(A) deletion in the $m t r R$ promoter region, G45D in the mtrR coding region, and A2047G (N. gonorrhoeae numbering, GenBank accession number: X67293.1) in 23S rRNA alleles (Table 2). Four allelic mutations in 23S rRNA were detected in two of three $(66.7 \%) N$. gonorrhoeae isolates exhibiting high-level AZM-R. Point mutations in $r p l D$ were identified in three isolates (G68D, G70D, and a combination of T69I and G70S). No mutations were detected in $r p l V$.

Thirteen strains were randomly selected from the 42 azithromycin-sensitive (AZM-S) strains as a control group. Mutations detected in these controls included: a single-nucleotide (A) deletion in the $m t r R$ promoter region and G45D in the $m t r R$ coding region in eight and three isolates, respectively (Table 2). No mutations were detected in the rplD and rplV genes or the 23S rRNA alleles in the AZM-S group.

Comparison between the AZM-R and AZM-S groups indicated that the mutation rate in the $m t r R$ coding region was not significantly different between them $(P>0.05)$, whereas there was a significant difference in the rate of mutation of the $m t r R$ promoter region between the two groups $(P<0.05)$.

\section{Molecular Epidemiologic Typing}

The $55 \mathrm{~N}$. gonorrhoeae clinical isolates were typed by NGMAST analysis and 43 different sequence types (STs) were identified, with $21(48.8 \%, 21 / 43)$ represented by only a single isolate. ST14781 was the most prevalent ST, accounting for four isolates, followed by ST1766 (three isolates), ST1866 (three isolates), ST5061 (three isolates), ST1407 (two isolates), ST4007 (two isolates), and ST4846 (two isolates). Fifteen of the STs $(34.9 \%, 15 / 43)$ identified in the present study have not been reported previously in the NGMAST database. Among the 13 AZM-R isolates, ST1866 was the most prevalent $(23.1 \%, 3 / 13)$, followed by ST1407 (15.4\%, 2/13). All three NG-MAST ST1866 isolates (which were not epidemiologically linked to one another) exhibited high-level AZM-R.

A total of 10 different STs, including four novel STs determined by MLST, were identified among the 13 AZM$\mathrm{R}$ isolates (Table 2), of which ST10,899 accounted for four isolates $(30.8 \%, 4 / 13)$. Three NG-MAST ST1866 isolates with high-level AZM-R belonged to MLST ST10899, indicating that they originated from the same clone.

Based on phylogenetic analysis, four large clusters (including $\geq 6$ isolates) were identified, which excluded 15 new-found types (Figure 1). Cluster A isolates included 


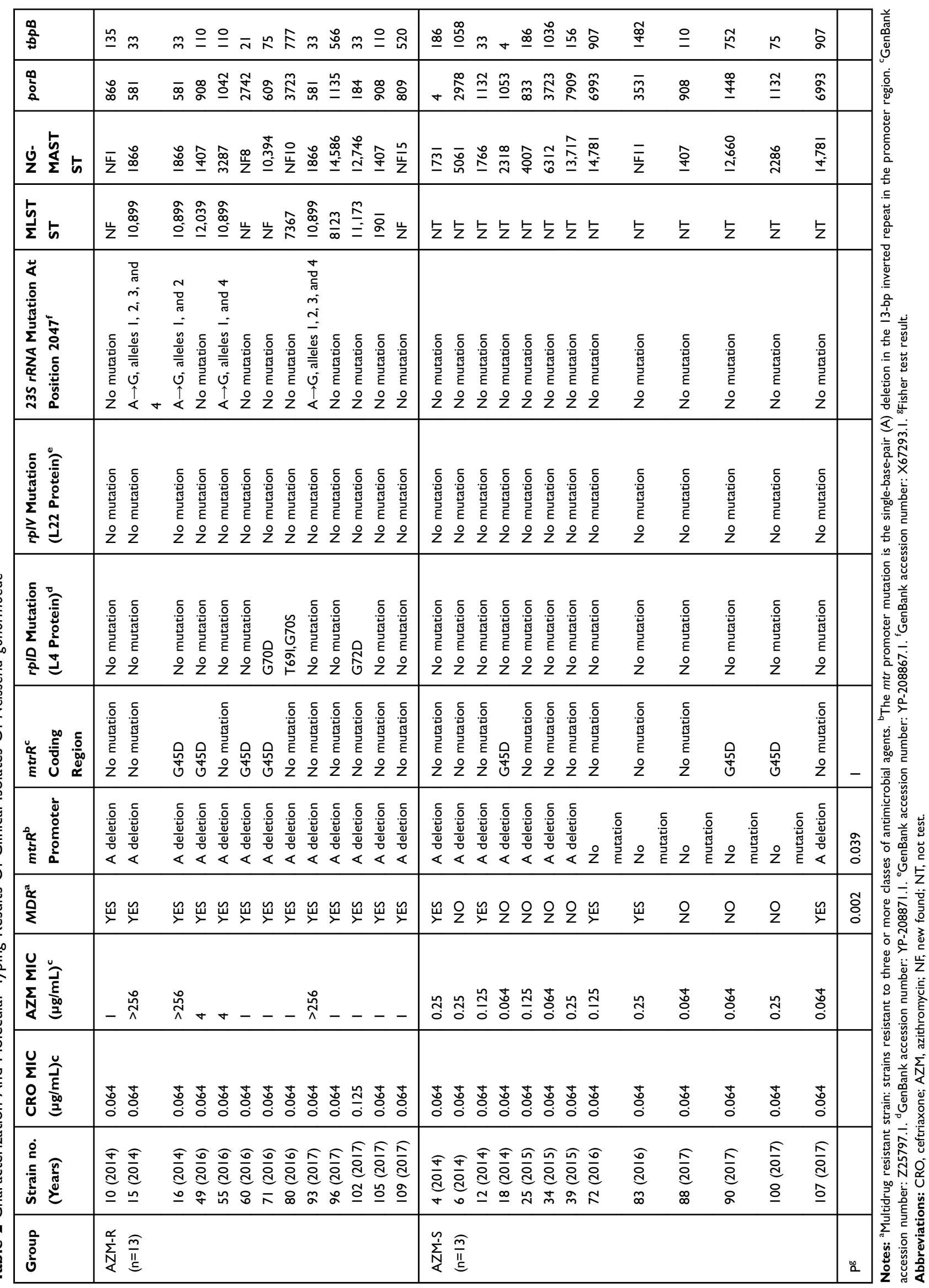




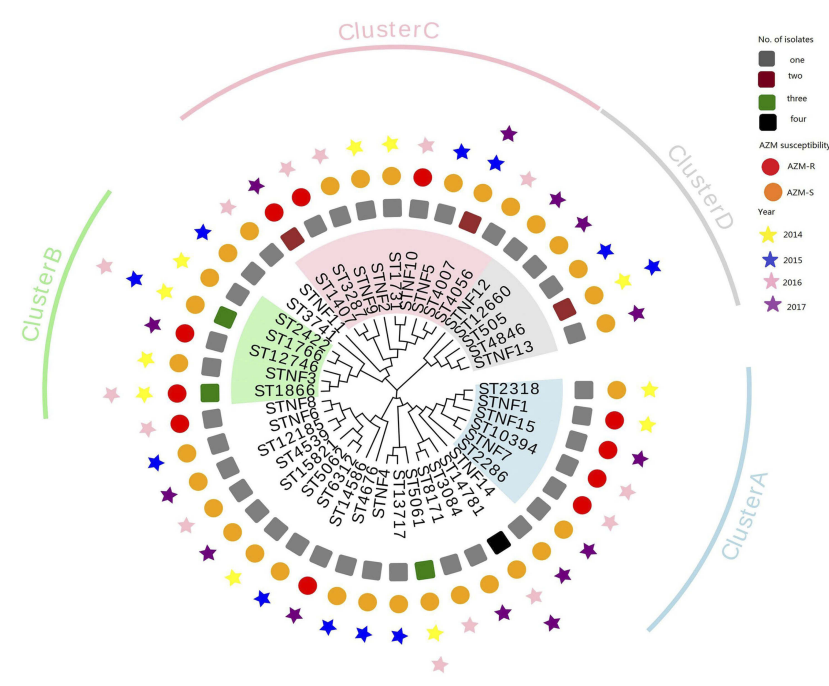

Figure I Phylogenetic tree constructed using MEGA7.0 for NG-MAST STs of $55 \mathrm{~N}$. gonorrhoeae isolates from Wenzhou, China, 2014 to 2017.

six different STs, half of them with $t b p B 75$ (ST2286, ST10394, and STNF7 isolates). Cluster B isolates included five different STs with $t b p B 186$ (ST2422) or $t b p B 33$ (ST1766, ST1866, ST12746, and STNF3). Cluster C isolates were represented by nine different STs (ST1407 and ST3287 isolates with $t b p B 110$ and ST1731, ST4007, ST4056, and STNF2 isolates with $t b p B$ 186). Finally, AZM-S cluster D contained five different STs, including ST505 and STNF12, both with porB 90; ST1660, ST4846, and STNF13 with porB 1448.

\section{Discussion}

Gonorrhea is the second most common sexually transmitted infection (STI) in the world and remains a major public health problem in China. From 2015 to 2016, the number of gonorrhea cases reported in China increased by $14.7 \%(100,245$ to 115,024$) .{ }^{38}$ In recent years, due to the overuse of antimicrobial agents (in China, it is common for doctors in small clinics to use antibiotics at will) and the evolution of bacteria, the resistance of $N$. gonorrhoeae to antibiotics has increased annually, ${ }^{21}$ bringing enormous challenges for the clinical treatment of $N$. gonorrhoeae infections. The resistance rates of $N$. gonorrhoeae to penicillin and ciprofloxacin in the present study (>90\%) were similar to those reported previously, ${ }^{11}$ indicating that these two antibacterial drugs should not be used for the treatment of gonorrhea. Resistance to tetracycline and azithromycin was $34.5 \%$ and $23.6 \%$ in our study; therefore, their use is not at all advisable (based on the WHO recommendations: "in general; an antibiotic should not be used when more than $5 \%$ of strains are resistant to it"; Tapsal 2001; WHO/CDS/CSR/DRS/2001.3). All strains were sensitive to spectinomycin and ceftriaxone in our study, which are widely used in China for the treatment of gonorrhea; hence these can still be used as first-line drugs to treat gonorrhea (in China, the extended-spectrum cephalosporin ceftriaxone has been recommended as a monotherapy for gonorrhea since $2007^{39}$ ). However, ceftriaxone-resistant $N$. gonorrhoeae has been detected in several studies; ${ }^{22-24}$ therefore, it is necessary to strengthen surveillance for ceftriaxone resistance.

In this study, the rate of AZM-R was $29.41 \%$ in both 2016 and 2017, similar to other recent reports from China. For example, analysis of 126 isolates from Hefei from 2014 to 2015 showed that 29\% were AZM-R, including $10 \%$ exhibiting high-level

AZM-R. ${ }^{17}$ Furthermore, a similar incidence was reported among 384 isolates from Nanjing during 20132014, with $32 \%$ of isolates displaying AZM-R and $10 \%$ showing high-level resistance. ${ }^{11}$ Notably, the levels of resistance to azithromycin observed in earlier studies from other regions in China were lower. ${ }^{25,26}$ As two neighboring countries in Asia, the resistance rates of $N$. gonorrhoeae isolated from China and Japan to azithromycin are much higher than those from Europe and America. $^{2,27-29}$ Antimicrobial resistant $N$. gonorrhoeae was first reported in East Asia, subsequently appearing in other parts of the world. ${ }^{30}$

Mutations in the promoter or coding sequence of the $m t r R$ gene in macrolide-resistant $N$. gonorrhoeae strains can result in decreased expression of the MtrR repressor, thereby upregulating the MtrCDE efflux pump. ${ }^{31}$ In gonococcal strains with a single-base-pair (A) deletion in the 13bp inverted repeat sequence of the $m t r R$ promoter region, $m t r R$ expression is abrogated, while levels of $m \operatorname{tr} C D E$ are elevated, most likely because of an increased binding affinity of RNA polymerase for $m \operatorname{tr} C D E .^{32}$ Missense mutations in the $m t r R$ gene, for example, a G45D mutation in the helix-turn-helix motif in the MtrR repressor, can reduce binding of the repressor to the $m \operatorname{tr} C D E$ promoter. ${ }^{7}$ In the present study, the mutation rates of the $m t r R$ promoter and coding regions in AZM-R isolates were $100.0 \%$ and $30.8 \%$, respectively. Compared with the AZM-S group, the mutation rate of the mtrR promoter region in the AZM-R group was significantly higher, while there was no significant difference in the mutation rate of the mtrR coding region. This is in accordance with previously reported findings indicating that mutations in the $m t r R$ promoter region play 
a more important role in the resistance of $N$. gonorrhoeae to azithromycin than alterations in the $m t r R$ coding region. ${ }^{12}$ The mutations A40D, A39T, H105Y, and $m t_{120}$ reported by other investigators ${ }^{11,12,17,33}$ were not found in our study.

Previous studies reported that one or more of the four alleles of the $r r l$ gene within domain $\mathrm{V}$ of $23 \mathrm{~S}$ rRNA are associated with AZM-R, including the mutations, A2143G and C2599T (corresponding to A2059G and C2611T in E. coli, respectively). ${ }^{34,35}$ The mutation A2047G (N. gonorrhoeae numbering) in domain $\mathrm{V}$ of detected in the present study in four AZM-R N. gonorrhoeae, but was not found in the AZM-S group. The numbers of each 23S rRNA allele in which the A2047G mutation occurred were 4, 4, 2 , and 2, respectively, and the corresponding MIC values were $256,256,256$, and $4 \mu \mathrm{g} / \mathrm{mL}$, respectively. It is worth noting that the $\mathrm{A} 2047 \mathrm{G}$ mutation was detected in all strains with an MIC value of $>256 \mu \mathrm{g} / \mathrm{mL}$; therefore, we believe that having multiple alleles of $\mathrm{A} 2047 \mathrm{G}$ is a major determinant of high-level azithromycin resistance.

Three $N$. gonorrhoeae isolates with MIC values for azithromycin of $1.0 \mu \mathrm{g} / \mathrm{mL}$ were found to harbor mutations in rplD (one isolate with G68D, one with G70D, and another with both T69I and G70S mutations). To our best knowledge, this is the first report of the mutations, G70D, T69I, and G70S, in rplD, while the G68D mutation has been described previously by Jacobsson. ${ }^{33}$ Therefore, mutations at positions 68-70 of the $N$. gonorrhoeae rplD gene appeared to be associated with low-to mid-level AZM resistance. The strains with $r p l D$ gene mutations belonged to clusters A and C, with NG-MAST classifications of ST10394, ST12746, and NF, indicating that $N$. gonorrhoeae strains with this mutation are genetically diverse.

$N$. gonorrhoeae typing methods, such as NG-MAST and MLST, assist in understanding of the spread of gonorrhea. In the present study, $34.9 \%(15 / 43)$ of STs determined by NGMAST were newly recognized, and $48.8 \%$ (21/43) of these STs were represented by only a single isolate, indicating that these $N$. gonorrhoeae clinical isolates exhibit considerable genetic diversity. According to the phylogenetic tree, AZM$\mathrm{R}$ strains are scattered throughout each year. Furthermore, phylogenetic analysis demonstrated that AZM-R isolates were widely divergent and did not belong to any specific clusters. This may be related to the large numbers of temporary residents in Wenzhou (according to the main data bulletin of Wenzhou City's sixth national census in 2010, the city's permanent population was $9,122,100$, of which the number of inflows from outside the city was 2,842,200, accounting for $31.16 \%$ of the city's permanent population), which may provide a greater opportunity for the emergence of the $N$. gonorrhoeae isolates with new and multiple NGMAST STs; however, all three isolates with high-level AZMR belonged to NG-MAST ST1866 and MLST ST10899, unlike those reported from other countries, such as Sweden (NG-MAST ST285, ST332, and ST8727); $;^{5}$ Wales, England, and the United States (NG-MAST ST649); ;6,37 and Argentina (NG-MAST ST696). ${ }^{4}$ It is worth mentioning that the high level of AZM-R N. gonorrhoeae found in this study has the same NA-MAST and MLST type as the high-level AZM-R N. gonorrhoeae found in the Liu YH report, ${ }^{40}$ which indicates that the spread of NG-MAST ST1866, MLST ST10899 N. gonorrhoeae has crossed the Taiwan Strait. Furthermore, the NG-MAST ST1866 clone has previously been described in Nanjing, Hangzhou, and Hefei, ${ }^{6,11,17}$ indicating that this clone with high-level AZM-R has spread in eastern China, which is a cause for concern. In addition, we also noted that the extensive drug-resistant (XDR) strain mentioned in the Jennison $\mathrm{AV}^{41}$ report is highly resistant to azithromycin while resistant to ceftriaxone. The XDR is the NG-MAST 16848 and MLST 12039, different from the high level of AZM-R $N$. gonorrhoeae found in our study. However, further analysis revealed that MLST 12039 and MLST 10899 have six identical housekeeping genes, which differ only in the pdhc allele. This may mean that high-level AZM-R N. gonorrhoeae is only one step away from the XDR strain. This must be a cause for concern.

\section{Conclusion}

Although the sample size of this study is limited, it is of great significance to study the resistance of $N$. gonorrhoeae to azithromycin in Wenzhou, eastern China. Measures should be implemented to monitor the spread of the NGMAST ST1866 and MLST ST10899 N. gonorrhoeae clones, which exhibit high-level AZM-R in eastern China.

\section{Ethics Approval}

Because the $N$. gonorrhoeae were part of the routine hospital laboratory procedure, ethics approval was not required.

\section{Acknowledgments}

The authors specially thank professor Yu Fangyou for his guidance and support. This work was supported by Wenzhou Municipal Science and Technology Bureau, China (No. Y20170808). 


\section{Author Contributions}

All authors contributed to data analysis, drafting or revising the article, gave final approval of the version to be published, and agree to be accountable for all aspects of the work.

\section{Disclosure}

The authors report no conflicts of interest in this work.

\section{References}

1. Newman L, Rowley J, Vander Hoorn S, et al. Global estimates of the prevalence and incidence of four curable sexually transmitted infections in 2012 based on systematic review and global reporting. PLoS One. 2015;10(12):e0143304. doi:10.1371/journal.pone.0143304

2. Kirkcaldy RD, Harvey A, Papp JR, et al. Neisseria gonorrhoeae antimicrobial susceptibility surveillance - the gonococcal isolate surveillance project, 27 sites, United States, 2014. MMWR Surveill Summ. 2016;65(7):1-19. doi:10.15585/mmwr.ss6507a1

3. Cole MJ, Spiteri G, Jacobsson S, et al. Overall low extended-spectrum cephalosporin resistance but high azithromycin resistance in neisseria gonorrhoeae in 24 European countries, 2015. BMC Infect Dis. 2017;17(1):617. doi:10.1186/s12879-017-2757-2

4. Galarza PG, Alcala B, Salcedo C, et al. Emergence of high level azithromycin-resistant Neisseria gonorrhoeae strain isolated in Argentina. Sex Transm Dis. 2009;36(12):787-788. doi:10.1097/ OLQ.0b013e3181b61bb1

5. Unemo M, Golparian D, Hellmark B. First three Neisseria gonorrhoeae isolates with high-level resistance to azithromycin in Sweden: a threat to currently available dual-antimicrobial regimens for treatment of gonorrhea? Antimicrob Agents Chemother. 2014;58(1):624625. doi:10.1128/AAC.02093-13

6. Ni C, Xue J, Zhang C, Zhou H, van der Veen S. High prevalence of Neisseria gonorrhoeae with high-level resistance to azithromycin in Hangzhou, China. J Antimicrob Chemother. 2016;71(8):2355-2357. doi:10.1093/jac/dkw131

7. Lucas CE, Balthazar JT, Hagman KE, Shafer WM. The MtrR repressor binds the DNA sequence between the mtrR and mtrC genes of Neisseria gonorrhoeae. $J$ Bacteriol. 1997;179(13):4123-4128. doi:10.1128/jb.179.13.4123-4128.1997

8. Luna VA, Cousin S Jr., Whittington WL, Roberts MC. Identification of the conjugative mef gene in clinical Acinetobacter junii and Neisseria gonorrhoeae isolates. Antimicrob Agents Chemother. 2000;44(9):2503-2506. doi:10.1128/aac.44.9.2503-2506.2000

9. Gregory ST, Dahlberg AE. Erythromycin resistance mutations in ribosomal proteins L22 and L4 perturb the higher order structure of $23 \mathrm{~S}$ ribosomal RNA. J Mol Biol. 1999;289(4):827-834. doi:10.1006/ jmbi.1999.2839

10. Tait-Kamradt A, Davies T, Cronan M, et al. Mutations in 23S rRNA and ribosomal protein L4 account for resistance in pneumococcal strains selected in vitro by macrolide passage. Antimicrob Agents Chemother. 2000;44(8):2118-2125. doi:10.1128/aac.44.8.2118-2125.2000

11. Wan C, Li Y, Le WJ, et al. Increasing resistance to azithromycin in neisseria gonorrhoeae in Eastern Chinese cities: resistance mechanisms and genetic diversity among isolates from Nanjing. Antimicrob Agents Chemother. 2018;62(5). doi:10.1128/AAC.02499-17

12. Demczuk W, Martin I, Peterson S, et al. Genomic epidemiology and molecular resistance mechanisms of azithromycin-resistant neisseria gonorrhoeae in Canada from 1997 to 2014. J Clin Microbiol. 2016;54 (5):1304-1313. doi:10.1128/JCM.03195-15

13. Mavroidi A, Tzelepi E, Siatravani E, et al. Analysis of emergence of quinolone-resistant gonococci in Greece by combined use of Neisseria gonorrhoeae multiantigen sequence typing and multilocus sequence typing. J Clin Microbiol. 2011;49(4):1196-1201. doi:10.1128/JCM.02233-10
14. Martin IM, Ison CA, Aanensen DM, Fenton KA, Spratt BG. Rapid sequence-based identification of gonococcal transmission clusters in a large metropolitan area. J Infect Dis. 2004;189(8):1497-1505. doi: $10.1086 / 383047$

15. Choudhury B, Risley CL, Ghani AC, et al. Identification of individuals with gonorrhoea within sexual networks: a population-based study. Lancet. 2006;368(9530):139-146. doi:10.1016/S0140-6736(06)69003-X

16. Liang JY, Cao WL, Li XD, et al. Azithromycin-resistant Neisseria gonorrhoeae isolates in Guangzhou, China (2009-2013): coevolution with decreased susceptibilities to ceftriaxone and genetic characteristics. BMC Infect Dis. 2016;16:152. doi:10.1186/s12879-016-1987-z

17. Jiang FX, Lan Q, Le WJ, Su XH. Antimicrobial susceptibility of Neisseria gonorrhoeae isolates from Hefei (2014-2015): genetic characteristics of antimicrobial resistance. BMC Infect Dis. 2017;17 (1):366. doi:10.1186/s12879-017-2757-2

18. Clinical and Laboratory Standards Institute. Performance Standards for Antimicrobial Susceptibility Testing. 29th ed.CLSI Supplement M100. Wayne (PA): CLSI; 2019.

19. Depardieu F, Courvalin P. Mutation in 23S rRNA responsible for resistance to 16-membered macrolides and streptogramins in Streptococcus pneumoniae. Antimicrob Agents Chemother. 2001;45 (1):319-323. doi:10.1128/AAC.45.1.319-323.2001

20. Allen VG, Farrell DJ, Rebbapragada A, et al. Molecular analysis of antimicrobial resistance mechanisms in Neisseria gonorrhoeae isolates from Ontario, Canada. Journal. 2011;55(Issue):703-712.

21. Ng LK, Martin I, Liu G, Bryden L. Mutation in $23 \mathrm{~S}$ rRNA associated with macrolide resistance in Neisseria gonorrhoeae. Antimicrob Agents Chemother. 2002;46(9):3020-3025. doi:10. 1128/aac.46.9.3020-3025.2002

22. Shimuta K, Watanabe Y, Nakayama S, et al. Emergence and evolution of internationally disseminated cephalosporin-resistant Neisseria gonorrhoeae clones from 1995 to 2005 in Japan. BMC Infect Dis. 2015;15:378. doi:10.1186/s12879-015-1110-x

23. Yin YP, Han Y, Dai XQ, et al. Susceptibility of Neisseria gonorrhoeae to azithromycin and ceftriaxone in China: a retrospective study of national surveillance data from 2013 to 2016. PLoS Med. 2018;15(2):e1002499. doi:10.1371/journal.pmed.1002593

24. Yan J, Xue J, Chen Y, et al. Increasing prevalence of Neisseria gonorrhoeae with decreased susceptibility to ceftriaxone and resistance to azithromycin in Hangzhou, China (2015-17). J Antimicrob Chemother. 2019;74(1):29-37. doi:10.1093/jac/dky412

25. Li W, Zhu BY, Qin SQ, et al. Surveillance of antibiotic susceptibility patterns of Neisseria gonorrhoeae from 2013 to 2015 in Guangxi Province, China. Jpn J Infect Dis. 2018;71(2):148-151. doi:10.7883/yoken.JJID.2017.169

26. Zhao L, Liu A, Li R, Zhao S. Trends in antimicrobial resistance in Neisseria gonorrhoeae and molecular characteristics of $\mathrm{N}$. gonorrhoeae with decreased susceptibility to ceftriaxone in Shandong, China, 2007 to 2014. Int J Antimicrob Agents. 2018;51(1):52-56. doi:10.1016/j.ijantimicag.2017.06.004

27. Lahra MM, Enriquez RP. Australian Gonococcal Surveillance Programme, 1 July to 30 September 2015. Commun Dis Intell $Q$ Rep. 2016;40(1):E179-81.

28. Yasuda M, Hatazaki K, Ito S, et al. Antimicrobial Susceptibility of Neisseria gonorrhoeae in Japan from 2000 to 2015. Sex Transm Dis. 2017;44(3):149-153. doi:10.1097/OLQ.0000000000000556

29. Gonococcal Antimicrobial Susceptibility Surveillance in EuropeResults summary 2017. European Centre for Disease Prevention and Control(ECDC).2019.doi:10.2900/415493.

30. Centers for Disease Control and Prevention (CDC). Cephalosporin susceptibility among Neisseria gonorrhoeae isolates-united States, 2000-2010. MMWR Morb Mortal Wkly Rep. 2011;60(26):873-877.

31. Lahra MM. Annual report of the Australian gonococcal surveillance programme, 2011. Commun Dis Intell Q Rep. 2012;36(2):E166-E173. 
32. Hagman KE, Shafer WM. Transcriptional control of the mtr efflux system of Neisseria gonorrhoeae. J Bacteriol. 1995;177(14):41624165. doi:10.1128/jb.177.14.4162-4165.1995

33. Jacobsson S, Golparian D, Cole M, et al. WGS analysis and molecular resistance mechanisms of azithromycin-resistant (MIC $>2 \mathrm{mg} / \mathrm{L}$ ) Neisseria gonorrhoeae isolates in Europe from 2009 to 2014. $J$ Antimicrob Chemother. 2016;71(11):3109-3116. doi:10.1093/jac/ dkw279

34. Allen VG, Seah C, Martin I, Melano RG. Azithromycin resistance is coevolving with reduced susceptibility to cephalosporins in Neisseria gonorrhoeae in Ontario, Canada. Antimicrob Agents Chemother. 2014;58(5):2528-2534. doi:10.1128/AAC.02608-13

35. Belkacem A, Jacquier H, Goubard A, et al. Molecular epidemiology and mechanisms of resistance of azithromycin-resistant Neisseria gonorrhoeae isolated in France during 2013-14. J Antimicrob Chemother. 2016;71(9):2471-2478. doi:10.1093/jac/dkw182

36. Katz AR, Komeya AY, Soge OO, et al. Neisseria gonorrhoeae with high-level resistance to azithromycin: case report of the first isolate identified in the United States. Clin Infect Dis. 2012;54(6):841-843. doi:10.1093/cid/cir929
37. Chisholm SA, Neal TJ, Alawattegama AB, et al. Emergence of high-level azithromycin resistance in Neisseria gonorrhoeae in England and Wales. $J$ Antimicrob Chemother. 2009;64(2):353-358. doi:10.1093/jac/dkp188

38. Epidemic of infectious diseases in China, 2016. Chinese Center for Disease Control and Prevention. 2018. http://www.nhfpc.gov.cn/jkj/ s3578/201702/38ca5990f8a54ddf9ca6308fec406157.shtml

39. Wang QQ, Zhang GC. Guidelines for Diagnosis and Treatment of Sexually Transmitted Diseases [In Chinese]. Shanghai: Shanghai Science and Technology Press; 2007.

40. Liu YH, Wang YH, Liao CH, Hsueh PR. Emergence and Spread of Neisseria gonorrhoeae Strains with High-level Resistance to Azithromycin in Taiwan from 2001 to 2018. Antimicrob Agents Chemother. 2019. doi:10.1128/AAC.00773-19

41. Jennison AV, Whiley D, Lahra MM, et al. Genetic relatedness of ceftriaxone-resistant and high-level azithromycin resistant Neisseria gonorrhoeae cases, United Kingdom and Australia, February to April 2018. Euro Surveill. 2019;24(8). doi:10.2807/1560-7917.ES.2019.24.8.1900118
Infection and Drug Resistance

\section{Publish your work in this journal}

Infection and Drug Resistance is an international, peer-reviewed openaccess journal that focuses on the optimal treatment of infection (bacterial, fungal and viral) and the development and institution of preventive strategies to minimize the development and spread of resistance. The journal is specifically concerned with the epidemiology of antibiotic resistance and the mechanisms of resistance development and diffusion in both hospitals and the community. The manuscript management system is completely online and includes a very quick and fair peerreview system, which is all easy to use. Visit http://www.dovepress.com/ testimonials.php to read real quotes from published authors. 